\title{
The fruit and vegetable intake of young Australian adults: a population perspective
}

\author{
Monica Nour ${ }^{1}{ }^{*}$, Zhixian Sui ${ }^{1}$, Amanda Grech ${ }^{1}$, Anna Rangan ${ }^{1}$, Kevin McGeechan ${ }^{2}$ and \\ Margaret Allman-Farinelli ${ }^{1}$ \\ 'The University of Sydney, School of Life and Environmental Sciences, Level 4 EAST, Charles Perkins Centre D17, \\ John Hopkins Drive, Sydney, NSW 2006, Australia: ${ }^{2}$ The University of Sydney, School of Public Health, Sydney, \\ NSW, Australia
}

Submitted 12 May 2016: Final revision received 18 April 2017: Accepted 5 May 2017: First published online 27 June 2017

\begin{abstract}
Objective: To examine intakes and variety of fruit and vegetables consumed by Australian young adults, also assessing differences by meal occasion and sociodemographic characteristics.

Design: Secondary analysis of cross-sectional $24 \mathrm{~h}$ recall data collected through the 2011-12 National Nutrition and Physical Activity Survey. Crude means and proportions consuming fruits and vegetables were calculated. Pearson $\chi^{2}$ tests, Kruskal-Wallis analyses and linear regression models were used to assess differences in mean intakes by age, BMI and sociodemographic variables. The variety eaten was determined based on the number of fruit and vegetable subgroups consumed.

Setting: Representative sample of metropolitan and rural areas across Australia. Subjects: Respondents aged 18-34 years were included ( $n$ 2397).

Results: Mean daily intake of fruit ( $128 \mathrm{~g} / 0.9$ servings) and vegetables (205 g/ $2 \cdot 7$ servings) was lower than the minimum recommended intake set at 2 and 5 servings, respectively. Age was positively associated with fruit and vegetable intake $(P=0.002, P<0.001)$, with $18-24$-year-olds reporting the poorest vegetable variety compared with 25-29- and 30-34-year-olds $(P=0.002)$. When controlling for total energy, males consumed less vegetables than females $(P=0 \cdot 002)$. A large proportion of the $15 \%$ of respondents who consumed adequate amounts of fruits and vegetables on the day prior to the survey reported intake across all meal occasions $(P<0 \cdot 001)$.

Conclusions: Fruit and vegetable intake is suboptimal among Australian young adults. An age-appropriate campaign is recommended to target increased consumption, particularly for those aged 18-24 years, with opportunity to promote increased variety and consumption across the day.
\end{abstract}

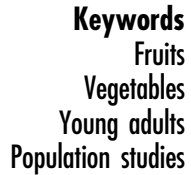

Fruits and vegetables are nutrient-dense foods, rich in fibre, vitamins, minerals and phytochemicals while being relatively low in energy. This makes them important components of a healthy diet. Regular consumption of an adequate intake is associated with lower risks of obesity ${ }^{(1)}$, cancers $^{(2-4)}$ CVD $^{(5-7)}$, stroke ${ }^{(8)}$, hypertension ${ }^{(9,10)}$ and allcause mortality $^{(11)}$. Guidelines vary by country, although most are consistent with the WHO's minimum recommendation of $400-500 \mathrm{~g}$ of fruits and vegetables daily (excluding potatoes and other starchy tubers) to reduce the risk of chronic disease ${ }^{(12-14)}$. In the UK, five daily portions of fruits and vegetables (combined weight of $400 \mathrm{~g}$ ) are recommended for health. This does not include starchy vegetables such as potatoes ${ }^{(14)}$. In Australia, two servings of fruits $(150 \mathrm{~g} /$ serving) and five servings of vegetables $(75 \mathrm{~g} /$ serving) are the minimum recommended daily intake for adults and include non-fried potatoes ${ }^{(15)}$. As these recommendations are based on gender-specific energy and nutrient requirements, adult males are recommended six servings of vegetables daily (total weight of $450 \mathrm{~g}$ ). Variety is also encouraged to maximise dietary diversity and the bioavailability of nutrients and other beneficial phytochemicals ${ }^{(15-18)}$.

Fruit and vegetable consumption levels are inadequate in many countries ${ }^{(19-23)}$. Internationally, the intake among young adults is particularly low ${ }^{(24,25)}$. Researchers and practitioners have made efforts to encourage intake and most recently the Australian government led the population-wide Go For $2 \& 5^{\circledR}$ campaign which resulted in a combined increase in consumption by 0.8 servings $/ \mathrm{d}^{(26)}$. 
Despite these efforts the latest statistics indicate that 19-30-year-old Australians are the poorest consumers of fruits and vegetables among adults ${ }^{(27)}$.

While formative research with young adults suggests that fruit and vegetable consumption is likely to increase during the transition to parenthood $^{(28,29)}$, if the pattern of suboptimal intake tracks into middle adulthood, it increases the risk of diet-related diseases among these adults and their offspring are likely to inherit these poor dietary patterns $^{(30)}$. Thus, innovative interventions and campaigns are needed to positively influence fruit and vegetable intake of future generations of adults. For maximum effect, interventions should be tailored to the target population ${ }^{(31)}$. This requires an in-depth understanding of the current patterns of intake and determinants of consumption.

The determinants of fruit and vegetable intake have been well documented in the literature, with gender, socioeconomic status (SES), personal preferences, availability and accessibility, and parental intake influencing consumption $^{(32)}$. Australian-wide studies specifically evaluating fruit and vegetable intake according to demographic associations are limited and more than 10 years old ${ }^{(33-35)}$, although there have been attempts to estimate intake at the state level such as the Western Australian report on intakes following the Go For $2 \& 5$ campaign $^{(26)}$. Prior to the most recent nutrition survey measuring food and dietary patterns (the 2011-12 National Nutrition and Physical Activity Survey (NNPAS)), the last national survey was conducted in $1995^{(36)}$. Preliminary results of the recent national survey show that fruit and vegetable intake remains inadequate ${ }^{(27)}$. However, this analysis does not account for all sources of fruits and vegetables in the diet. Detailed secondary analysis including mixed dishes where fruits and vegetables make a minor contribution might yield more complete results.

In 1995, Australians living in areas of lower SES with low incomes had the lowest fruit and vegetable intakes ${ }^{(35)}$. Previous literature has also demonstrated that access to fresh fruits and vegetables varies with geographical location $^{(37-39)}$. Other research has shown that increased vegetable intake can mediate weight loss in young adults $^{(40)}$. To provide context for interventions, current relationships between intake, sociodemographic variables, and factors such as BMI should be examined.

Dietary guidelines based on epidemiological evidence recommend consumption of a variety of fruits and vegetables to maximise bioavailability of nutrients including phytochemicals and the unique health benefits they confer $^{(15-17)}$. Thus, variety should be considered when planning interventions. Lastly, with recommendations set at five vegetable servings daily, it is unlikely that an individual will meet his/her requirements if vegetable consumption occurs in a single eating occasion. Thus, assessing distribution of intake across meal occasions is also of interest to discern opportunities for increased consumption.

Evaluating fruit and vegetable intake according to group characteristics and demographics can inform policy and health promotion practice to improve consumption levels. Thus, the present study aimed to conduct secondary analyses on the NNPAS data from 2011-12 in order to: (i) determine the intakes of fruits and vegetables among young adults (18-34-year-olds); (ii) evaluate variety of fruits and vegetables in the diets of young adults; (iii) investigate fruit and vegetable intakes by meal occasion (main meals $v$. snacks); and (iv) examine intakes according to sociodemographic variables such as age, gender, BMI, Socio-Economic Index for Areas (SEIFA) and geographical location.

\section{Methods}

\section{Participants and dietary data methodology}

The data analysed in the present study were collected as part of the 2011-12 Australian NNPAS by the Australian Bureau of Statistics (ABS). A detailed description of the survey methods including data collection and handling is available from the $\mathrm{ABS}^{(41)}$. Briefly, the 2011-12 NNPAS was conducted using nationally representative subsamples of the Australian Health Survey 2011-13. Trained ABS technicians collected dietary data on foods and beverages consumed using a computer-assisted personal interview, multiple-pass $24 \mathrm{~h}$ dietary recall. This method captured intakes of foods and beverages consumed by respondents on the day prior to the interview. To account for variations in intakes across seasons and days of the week, surveys were conducted over 12 months covering weekdays and weekends. Portion sizes were assessed by quantifying the amount of food the respondent consumed at one meal occasion. Rulers, rings, a grid, a wedge, various meat cuts and Australian-sourced drawings and photographs of actual-size food and drink containers in different shapes and sizes were provided in a food model booklet to help respondents estimate portion sizes, which were converted to grams by multiplying the volume specified by the food density ${ }^{(41)}$. A second $24 \mathrm{~h}$ recall was conducted with all participants asked to participate on a voluntary basis. Data from the second interview (computer-assisted telephone interview) was not included as only $64 \%$ of respondents participated in the second dietary recall. The survey included a representative sample of city, metropolitan, rural and remote areas across the Australian States and Territories. In the present paper, secondary analyses were conducted on fruit and vegetable intake data of young adults aged 18-34 years. This age range was chosen to reflect definitions of young adulthood according to national health institutes in the USA and Australia $^{(42,43)}$. However, as emerging adults may have quite different lifestyles from those aged $30-35$ years ${ }^{(44)}$, we further grouped into the following age categories: 18-24 years, 25-29 years and 30-34 years. Data were extracted from the Confidentialised Unit Record Files provided by the ABS (permission granted for use) ${ }^{(45)}$. 


\section{Classification of fruits and vegetables}

The Confidentialised Unit Record Files group food data for all respondents into categories. Further grouping was conducted to classify fruits and vegetables according to categories based on the foundation and total diet food models developed for dietary guidelines ${ }^{(18)}$. Fruits were categorised as citrus, pome, tropical, berries, stone or other; with a separate fruit juice category. Vegetables were grouped as green and brassica, orange, starchy, root/ tubular/bulb or other, excluding fried potatoes. Legumes, fresh, canned, frozen and dried varieties of fruits and vegetables, as well as fruits and vegetables within mixed dishes were included in the analyses (see online supplementary material, Table S1). All fruits and vegetables in mixed dishes were included. The proportions of fruits and vegetables within all mixed dishes were determined based on ingredient weights reported within the 2011-13 AUSNUT food recipe file ${ }^{(46)}$ and assigned to the appropriate fruit or vegetable category. Consumption of fruit juice exceeding $125 \mathrm{ml}$ and fried potatoes were excluded from analyses in accordance with the Australian Guide to Healthy Eating recommendations which classify them as discretionary (non-core) items ${ }^{(15)}$. Fried potato intake was assessed and reported separately.

\section{Assessment of fruit and vegetable intake}

The total weight of fruits and vegetables consumed by each respondent was calculated as the sum of the fruit and vegetable categories, which included both individual fruits and vegetables and those from mixed dishes. Consumers and non-consumers were identified and proportions were established. The mean intakes of fruits and vegetables (grams) were calculated and converted to servings. Internationally there is variation in the definition of a serving. For example, in the UK, a serving of fruit or vegetables is equivalent to $80 \mathrm{~g}^{(47)}$. We used the Australian Guide to Healthy Eating ${ }^{(15)}$ definition which specifies that a standard serving of fruit is equivalent to $150 \mathrm{~g}$, while a serving of vegetables equates to $75 \mathrm{~g}$, with a minimum of two servings of fruit and five servings of vegetables recommended daily for adults. These recommendations are based on gender-specific energy and nutrients requirements, such that adult males are recommended six servings of vegetables daily.

\section{Variety and intake by meal occasion}

The variety of fruits and vegetables eaten was calculated as the number of the fruit and vegetable categories consumed as defined in the online supplementary material, Table S1. Variety was assessed using a modified version of the scoring system developed by Magarey et al. ${ }^{(34)}$. Scoring was as follows: low variety (one type of fruit, one or two types of vegetable), medium variety (two types of fruit, three or four types of vegetable) and high variety (three or more types of fruit, five or more types of vegetable). For this analysis, consuming $\geq 50 \%$ of a serving of a category of fruit or vegetable as defined in Table S1 (i.e. $\geq 75 \mathrm{~g}$ of fruit or $\geq 37.5 \mathrm{~g}$ of vegetables) was counted as consuming one type of fruit or vegetable. The number of different types consumed by each participant was summed to give his/her total variety score. Fruit juice was excluded from variety scoring as the type of fruit within these products was not differentiated as part of the current analyses. Dried fruit was also excluded as only a small proportion of the population reported consumption on the day prior to the dietary recall. Data were also categorised by meal occasion as breakfast, lunch, dinner or snacks, where snacks included brunch, morning tea, afternoon tea, snack, extended consumption and other. The mean fruit and vegetable intake at each meal occasion was determined. Further analyses were conducted to explore patterns in number of servings consumed across the day and proportions consuming fruits and vegetables per meal occasion.

\section{Associations between fruit and vegetable intake and lifestyle, anthropometry and sociodemographic variables}

To explore factors that may influence fruit and vegetable consumption, we evaluated the relationship between age, BMI, sociodemographic variables (SEIFA and geographical location), lifestyle factors and mean intakes. BMI was derived from the height and weight measurements taken objectively by the interviewer and categorised as underweight $\left(\leq 18.5 \mathrm{~kg} / \mathrm{m}^{2}\right)$, healthy weight $\left(18.5-24.99 \mathrm{~kg} / \mathrm{m}^{2}\right)$, overweight $\left(25 \cdot 0-29 \cdot 99 \mathrm{~kg} / \mathrm{m}^{2}\right)$ or obese $\left(\geq 30.0 \mathrm{~kg} / \mathrm{m}^{2}\right)$ based on the National Institutes of Health's cut-offs ${ }^{(48)}$. Respondents with no BMI recording ( $n$ 317) were coded as 'missing values' and omitted from BMI analyses. The SEIFA takes into consideration the impact of the area of residence, rather than an individual's income, occupation or level of education, on intake. Quintile 1 includes the most disadvantaged areas, while quintile 5 represents the least disadvantaged areas. Geographical location was categorised as inner regional Australia, city/metropolitan (capital cities and surrounds) and other (outer regional Australia, remote and very remote Australia). Data on smoking (smoker $v$. non-smoker) and alcohol consumption (grams per day) were also evaluated as potential confounders in regression models.

\section{Statistics}

Statistical analyses were conducted using the statistical software package IBM SPSS Statistics for Windows version 22.0. Data for those aged 18-34 years inclusive were extracted from the Confidentialised Unit Record Files. Subject weighting factors supplied by the ABS were applied to the data before analyses, to ensure they were more representative of the population by age, gender, area of residence and seasonal effect ${ }^{(41)}$. Under-reporters were identified as those with a ratio of energy intake to BMR of $<0.87$ based on the Goldberg cut-off ${ }^{(49)}$, which has been 
used for identification of misreporting in previous national Australian surveys ${ }^{(50)}$ and validated for use with $24 \mathrm{~h}$ recall data ${ }^{(51)}$. Results are reported including under-reporters unless stated otherwise. Descriptive statistics were used to report fruit and vegetable intake. The mean intake per capita and median intake per consumer were determined and percentage consuming calculated. Differences in proportions of young adults consuming fruits and vegetables according gender, age, BMI, SEIFA and geographical location were assessed using Pearson's $\chi^{2}$ tests. Differences in variety scores and proportions of persons consuming vegetables at each meal occasion according to categories of servings consumed were also determined by Pearson $\chi^{2}$ tests. As data were not normally distributed, Kruskal-Wallis tests were applied to assess trends in intakes across categories and by age and gender, and to compare differences in intakes between meal occasions. Linear regression models were used to determine the relationship between fruit and vegetable intake and age, gender, BMI and sociodemographic variables (SEIFA and geographical location), controlling for energy intake and lifestyle factors (smoking status and alcohol intake). Statistical significance was set at $P<0.05$ for all tests.

\section{Results}

\section{Characteristics}

Table 1 summarises the characteristics of the sample of young adults included within the analyses ( $n$ 2397). The sample was evenly distributed across genders, age and SEIFA. Close to half the population were classed as overweight or obese (Table 1). Approximately $16 \%$ of respondents were classed as under-reporters ( $n$ 386).

\section{Proportions of young adults consuming fruits and vegetables}

Proportions of young adults consuming fruits and vegetables, and the amounts consumed, according to age, gender, BMI, SEIFA and geographical location, are presented in Tables 2-5. Fifty-six per cent of respondents consumed fruit (48\% when excluding fruit juice) and $93 \%$ consumed vegetables. A small percentage of respondents ( $4.3 \%$ ) did not consume any fruit or vegetables. A greater proportion of females consumed fruits than males (males, $40.6 \%$; females, $53.8 \% ; P<0 \cdot 001$ ). No significant differences were observed between genders for vegetable consumption (Table 2). Fewer young adults aged 18-24 years reported consuming fruits (Table 4), and the largest percentage of consumers was observed in the young adults of the highest SEIFA category for fruit when including juice (Table 5) and for vegetables (Table 3). The proportion consuming legumes on the day prior to the dietary recall was relatively low at $12.3 \%$. Pome fruit and fruit juice were the most popular fruit categories consumed (Table 4).
Table 1 Characteristics of the sample of Australian young adults from the National Nutrition and Physical Activity Survey 2011-12 ( $n$ 2397)

\begin{tabular}{|c|c|c|}
\hline Characteristic & $\%$ & $n$ \\
\hline \multicolumn{3}{|l|}{ Sex } \\
\hline Male & $46 \cdot 7$ & 1120 \\
\hline Female & $53 \cdot 3$ & 1277 \\
\hline \multicolumn{3}{|l|}{ Age (years) } \\
\hline $18-24$ & 32.5 & 780 \\
\hline $25-29$ & 30.7 & 736 \\
\hline 30-34 & $36 \cdot 8$ & 881 \\
\hline \multicolumn{3}{|c|}{ Socio-Economic Index for Areas (SEIFA) } \\
\hline Lowest $20 \%$ & $18 \cdot 8$ & 451 \\
\hline Second quintile & $20 \cdot 8$ & 499 \\
\hline Third quintile & $20 \cdot 4$ & 490 \\
\hline Fourth quintile & $17 \cdot 5$ & 419 \\
\hline Highest $20 \%$ & $22 \cdot 4$ & 538 \\
\hline \multicolumn{3}{|l|}{ Geographical location } \\
\hline City & $69 \cdot 0$ & 1654 \\
\hline Inner regional & $17 \cdot 0$ & 408 \\
\hline Outer regional/remote & $14 \cdot 0$ & 335 \\
\hline \multicolumn{3}{|l|}{ BMI $\left(\mathrm{kg} / \mathrm{m}^{2}\right)^{\star}$} \\
\hline Underweight $(<18.5)$ & $3 \cdot 2$ & 67 \\
\hline Healthy weight (18.5-24.99) & $47 \cdot 1$ & 979 \\
\hline Overweight (25.0-29.99) & $32 \cdot 2$ & 669 \\
\hline Obese $(\geq 30 \cdot 0)$ & $17 \cdot 5$ & 365 \\
\hline \multicolumn{3}{|l|}{ Currently smoking } \\
\hline Yes & 22.7 & 554 \\
\hline No & $77 \cdot 3$ & 1854 \\
\hline \multicolumn{3}{|c|}{ Consumed alcohol on the day surveyed } \\
\hline Yes & $26 \cdot 2$ & 629 \\
\hline No & 73.8 & 1768 \\
\hline
\end{tabular}

\section{Amounts of fruits and vegetables consumed}

Median intake among consumers was 181.5 and $159.5 \mathrm{~g}$ for fruit and vegetables, respectively. This is equivalent to $1 \cdot 2$ servings of fruit and $2 \cdot 1$ servings of vegetables using Australian standard serving sizes. The median (interquartile range; 25 th-75th percentile) intake of fried potatoes among 18-34-year-olds was 88.5 (55.0-134.3) g, which, if included, would bring the median servings of vegetables consumed to 3.3 servings. Intake of vegetables was lowest for 18-24-year-olds ( $P=0.002$; Table 2$)$. Fruit intake (including juice) was highest for the 30-34-yearolds $(P=0 \cdot 002)$, with females consuming more than males $(P<0.001 ;$ Table 4$)$. Those within the obese category reported the lowest intake of fruits $(P=0.02$; Table 4$)$. While no significant differences were found between SEIFA quintiles for vegetable intake, consumption patterns were trending towards significance $(P=0 \cdot 06)$. Geographical location had no significant effect on vegetable intake. However, those within regional locations reported consuming more starchy vegetables $(P<0 \cdot 001)$ and less of the 'others' category $(P=0 \cdot 045$; Table 3$)$.

\section{Comparison of per capita intake with Australian Guide to Healthy Eating recommendations}

On average, 18-34-year-olds consumed $128 \mathrm{~g}$ (0.9 servings) of fruit, which was below the $300 \mathrm{~g}$ ( 2 servings) minimum daily recommendation. The mean vegetable intake was $205 \mathrm{~g}$ ( 2.7 servings), also below the $375 \mathrm{~g}$ 


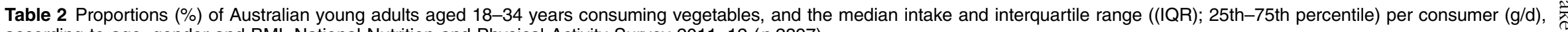
according to age, gender and BMI, National Nutrition and Physical Activity Survey 2011-12 (n 2397)

\begin{tabular}{|c|c|c|c|c|c|c|c|c|c|c|c|c|c|c|c|c|c|}
\hline & \multicolumn{7}{|c|}{ Gender } & \multicolumn{10}{|c|}{ Age group (years) } \\
\hline & \multicolumn{3}{|c|}{ Male } & \multicolumn{3}{|c|}{ Female } & \multirow[b]{2}{*}{$P^{*}$} & \multicolumn{3}{|c|}{$18-24$} & \multicolumn{3}{|c|}{$25-29$} & \multicolumn{3}{|c|}{$30-34$} & \multirow[b]{2}{*}{$P^{*}$} \\
\hline & $\%$ & Median & IQR & $\%$ & Median & IQR & & $\%$ & Median & IQR & $\%$ & Median & IQR & $\%$ & Median & IQR & \\
\hline Total Vegf & 92.5 & 159 & $79 \cdot 0-299$ & 93.8 & 160 & $86 \cdot 4-284$ & 0.95 & 92.1 & 151 & $70 \cdot 8-270$ & 95.0 & 166 & $91 \cdot 7-308$ & $92 \cdot 7$ & 163 & $89 \cdot 6-306$ & 0.002 \\
\hline Green Veg & 72.9 & 28.9 & $12 \cdot 3-73 \cdot 8$ & 72.6 & 30.0 & $12 \cdot 5-70 \cdot 3$ & 0.97 & 69.9 & $26 \cdot 7$ & $8 \cdot 3-65 \cdot 0$ & $75 \cdot 8$ & 33.0 & $15 \cdot 0-74 \cdot 0$ & $72 \cdot 8$ & 31.0 & $12.5-74.0$ & 0.002 \\
\hline Legumes & 12.5 & $44 \cdot 8$ & $8 \cdot 5-148$ & $12 \cdot 1$ & 38.6 & $6 \cdot 8-137$ & 0.70 & $10 \cdot 6$ & $26 \cdot 4$ & $4 \cdot 5-120$ & 13.0 & $50 \cdot 0$ & $8 \cdot 7-138$ & $13 \cdot 1$ & $44 \cdot 0$ & $13 \cdot 4-140$ & 0.24 \\
\hline Orange Veg & 33.8 & $30 \cdot 0$ & $14 \cdot 0-66 \cdot 6$ & $36 \cdot 3$ & 37.2 & $17 \cdot 8-70 \cdot 4$ & 0.09 & 33.1 & $33 \cdot 6$ & $15 \cdot 0-62 \cdot 8$ & 37.8 & 35.9 & $20.0-72.5$ & 34.7 & $32 \cdot 3$ & $14.0-68.8$ & 0.07 \\
\hline Root Veg & 69.4 & 21.4 & $9 \cdot 3-40 \cdot 8$ & $66 \cdot 2$ & 19.8 & $9 \cdot 2-37.5$ & 0.004 & 66.9 & 19.5 & $8.4-39.9$ & 69.4 & 21.6 & $9 \cdot 4-39 \cdot 0$ & $67 \cdot 0$ & $19 \cdot 8$ & $9.4-39.8$ & 0.61 \\
\hline Other Veg & $72 \cdot 6$ & $72 \cdot 0$ & $30 \cdot 6-125$ & $75 \cdot 3$ & $62 \cdot 4$ & $29 \cdot 4-123$ & 0.82 & $71 \cdot 2$ & $61 \cdot 8$ & $29 \cdot 0-115$ & $76 \cdot 9$ & 67.4 & $31 \cdot 7-132$ & 74.2 & $74 \cdot 0$ & $30 \cdot 0-128$ & 0.004 \\
\hline Starchy Veg & 27.5 & $89 \cdot 1$ & $26 \cdot 2-203$ & $34 \cdot 1$ & 89.1 & $32 \cdot 9-156$ & 0.001 & 28.5 & 103 & $40 \cdot 7-172$ & 32.5 & 78.0 & $21 \cdot 5-193$ & $32 \cdot 1$ & 82.5 & $26 \cdot 2-190$ & 0.27 \\
\hline
\end{tabular}

BMl $\left(\mathrm{kg} / \mathrm{m}^{2}\right) \dagger$

\begin{tabular}{|c|c|c|c|c|c|c|c|c|c|c|c|c|c|}
\hline & \multicolumn{3}{|c|}{$<18.5$} & \multicolumn{3}{|c|}{$18.5-24.99$} & \multicolumn{3}{|c|}{ 25.0-29.99 } & \multicolumn{3}{|c|}{$\geq 30.0$} & \multirow[b]{2}{*}{$P^{*}$} \\
\hline & $\%$ & Median & IQR & $\%$ & Median & IQR & $\%$ & Median & IQR & $\%$ & Median & IQR & \\
\hline Total Veg $\ddagger$ & 95.5 & 155 & $110-241$ & $94 \cdot 2$ & 161 & $82 \cdot 2-306$ & 93.0 & 158 & $90 \cdot 6-304$ & 91.2 & 159 & $68 \cdot 1-304$ & 0.36 \\
\hline Green Veg & 67.2 & 21.2 & $7 \cdot 6-60 \cdot 1$ & 73.0 & 28.9 & $12 \cdot 3-63 \cdot 3$ & 74.0 & 32.5 & $13.0-80.2$ & 70.7 & 28.8 & $15 \cdot 0-73 \cdot 4$ & 0.80 \\
\hline Legumes & 14.9 & $46 \cdot 4$ & $13 \cdot 4-740$ & $13 \cdot 6$ & $46 \cdot 0$ & $14 \cdot 8-100$ & $11 \cdot 2$ & 38.7 & $7 \cdot 9-138$ & $8 \cdot 8$ & 8.5 & $5 \cdot 1-37 \cdot 9$ & 0.06 \\
\hline Orange Veg & 31.3 & 45.4 & $25.0-71.9$ & 35.1 & $34 \cdot 1$ & $16 \cdot 7-75 \cdot 0$ & $35 \cdot 1$ & 34.5 & $16 \cdot 7-70 \cdot 0$ & $36 \cdot 2$ & 28.5 & $14.0-70 \cdot 2$ & 0.97 \\
\hline Root Veg & $68 \cdot 7$ & $16 \cdot 7$ & $10.7-57.9$ & 68.1 & $24 \cdot 1$ & $10 \cdot 0-41 \cdot 6$ & $66 \cdot 8$ & $17 \cdot 7$ & $8 \cdot 0-40 \cdot 0$ & 67.9 & $16 \cdot 3$ & $7 \cdot 5-36.5$ & 0.27 \\
\hline Other Veg & 71.6 & $70 \cdot 0$ & $39.4-110$ & 76.0 & 64.5 & $31 \cdot 3-131$ & $72 \cdot 9$ & 74.9 & $34 \cdot 8-124$ & 72.9 & 58.5 & $26 \cdot 0-115$ & 0.11 \\
\hline Starchy Veg & 31.3 & $97 \cdot 3$ & $43 \cdot 4-137$ & 31.2 & $96 \cdot 8$ & $28 \cdot 3-187$ & 30.5 & 92.5 & $24 \cdot 2-203$ & 31.8 & 82.5 & $19 \cdot 2-203$ & 0.92 \\
\hline
\end{tabular}

*From Kruskal-Wallis test on per capita intakes; significant $P$ values indicated in bold font.

tn 2080, as 317 participants did not have a measured weight and height for calculation of BMl values.

fExcluding fried potatoes. 
Table 3 Proportions (\%) of Australian young adults aged 18-34 years consuming vegetables, and the median intake and interquartile range ((IQR); 25th-75th percentile) per consumer ( $\mathrm{g} / \mathrm{d})$, according to Socio-Economic Index for Areas (SEIFA) and geographical location, National Nutrition and Physical Activity Survey 2011-12 ( $n$ 2397)

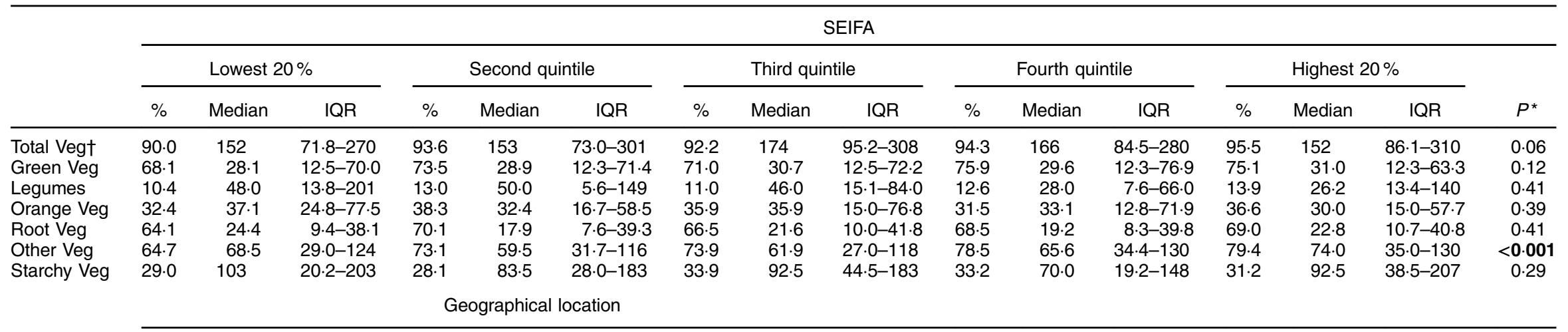

\begin{tabular}{|c|c|c|c|c|c|c|c|c|c|c|}
\hline & \multicolumn{3}{|c|}{ City } & \multicolumn{3}{|c|}{ Inner Regional } & \multicolumn{3}{|c|}{ Outer regional/remote } & \multirow[b]{2}{*}{$P^{*}$} \\
\hline & $\%$ & Median & IQR & $\%$ & Median & IQR & $\%$ & Median & IQR & \\
\hline Total Vegt & 93.5 & 158 & $82 \cdot 4-279$ & 92.9 & 188 & $92 \cdot 0-330$ & 92.2 & 133 & $73 \cdot 0-294$ & 0.15 \\
\hline Green Veg & 73.8 & 28.7 & $12 \cdot 3-70 \cdot 8$ & $71 \cdot 1$ & 31.8 & $15 \cdot 0-76 \cdot 3$ & 69.6 & 38.4 & $17.0-70.0$ & 0.75 \\
\hline Legumes & $12 \cdot 6$ & $44 \cdot 8$ & $13 \cdot 4-140$ & $12 \cdot 0$ & $22 \cdot 0$ & $4 \cdot 2-120$ & 11.0 & 49.7 & $6 \cdot 8-138$ & 0.71 \\
\hline Orange Veg & 34.2 & 32.8 & $15 \cdot 5-67 \cdot 0$ & 37.0 & 40.5 & $26 \cdot 0-70 \cdot 2$ & $37 \cdot 3$ & $26 \cdot 0$ & $14.0-68.8$ & 0.30 \\
\hline Root Veg & 68.6 & 20.5 & $9 \cdot 4-40 \cdot 8$ & 66.7 & 20.4 & $7.1-35.0$ & 64.8 & $16 \cdot 3$ & $8 \cdot 3-34.6$ & 0.31 \\
\hline Other Veg & $75 \cdot 8$ & $67 \cdot 1$ & $30 \cdot 6-126$ & 68.9 & $62 \cdot 4$ & $29 \cdot 0-124$ & 71.9 & $61 \cdot 8$ & $29 \cdot 0-107$ & 0.045 \\
\hline Starchy Veg & $30 \cdot 1$ & $80 \cdot 8$ & $25 \cdot 7-168$ & 38.2 & 110 & $51.9-196$ & 26.9 & 107 & $46 \cdot 6-193$ & $<0.001$ \\
\hline
\end{tabular}

${ }^{*}$ From Kruskal-Wallis test on per capita intakes; significant $P$ values indicated in bold font.

tExcluding fried potatoes. 
Table 4 Proportions (\%) of Australian young adults aged 18-34 years consuming fruit, and the median intake and interquartile range ((IQR); 25th-75th percentile) per consumer (g/d), according to age, gender and BMI, National Nutrition and Physical Activity Survey 2011-12 ( $n$ 2397)

\begin{tabular}{|c|c|c|c|c|c|c|c|c|c|c|c|c|c|c|c|c|c|}
\hline & \multicolumn{7}{|c|}{ Gender } & \multicolumn{10}{|c|}{ Age group (years) } \\
\hline & \multicolumn{3}{|c|}{ Male } & \multicolumn{3}{|c|}{ Female } & \multirow[b]{2}{*}{$P^{*}$} & \multicolumn{3}{|c|}{$18-24$} & \multicolumn{3}{|c|}{$25-29$} & \multicolumn{3}{|c|}{$30-34$} & \multirow[b]{2}{*}{$P^{*}$} \\
\hline & $\%$ & Median & IQR & $\%$ & Median & IQR & & $\%$ & Median & IQR & $\%$ & Median & IQR & $\%$ & Median & IQR & \\
\hline Total Fruit without Juice & 40.6 & 188 & $114-359$ & 53.8 & 175 & 103-262 & $<0.001$ & $41 \cdot 8$ & 184 & 114-309 & $49 \cdot 0$ & 175 & $90-263$ & 51.6 & 184 & $131-300$ & 0.002 \\
\hline Total Fruit including Juiceł & 50.8 & 164 & $150-314$ & $59 \cdot 4$ & 178 & $139-298$ & $<0.001$ & $49 \cdot 6$ & 166 & $150-310$ & $57 \cdot 3$ & 167 & $150-295$ & $58 \cdot 3$ & 175 & $150-304$ & 0.002 \\
\hline Citrus Fruit & $10 \cdot 3$ & 131 & $75 \cdot 0-193$ & $12 \cdot 0$ & 93.0 & $75 \cdot 0-150$ & 0.21 & 9.7 & 131 & $65 \cdot 5-193$ & $12 \cdot 4$ & 75.0 & $75 \cdot 0-150$ & 11.5 & 131 & $75 \cdot 0-150$ & 0.23 \\
\hline Pome Fruit & 20.8 & 173 & 164-196 & $25 \cdot 1$ & 164 & $143-188$ & 0.045 & 21.3 & 164 & $143-188$ & 23.0 & 164 & $158-188$ & 24.7 & 164 & $164-188$ & 0.23 \\
\hline Tropical Fruit & $7 \cdot 2$ & 55.5 & $18 \cdot 0-245$ & $9 \cdot 2$ & $45 \cdot 0$ & $18 \cdot 5-112$ & 0.08 & 9.6 & 51.0 & $21 \cdot 2-184$ & 7.6 & $45 \cdot 0$ & $10 \cdot 5-159$ & $7 \cdot 7$ & 44.3 & $18 \cdot 0-184$ & 0.27 \\
\hline Berries & 4.7 & $44 \cdot 3$ & $24 \cdot 0-124$ & 9.8 & 38.5 & $24 \cdot 0-70 \cdot 1$ & 0.001 & $6 \cdot 4$ & 35.2 & $19 \cdot 0-110$ & 7.2 & 57.7 & $24 \cdot 0-135$ & 8.5 & 41.9 & $24 \cdot 0-80 \cdot 0$ & 0.28 \\
\hline Stone Fruit & $5 \cdot 2$ & 151 & $66 \cdot 0-295$ & $7 \cdot 4$ & 145 & $54 \cdot 0-151$ & 0.03 & $5 \cdot 8$ & 140 & $23 \cdot 3-166$ & $5 \cdot \overline{7}$ & 145 & $47 \cdot 3-175$ & 7.5 & 151 & $109-288$ & 0.23 \\
\hline Other Fruit & 9.3 & $85 \cdot 8$ & $27 \cdot 0-156$ & $16 \cdot 1$ & 78.0 & $40 \cdot 0-175$ & $<0.001$ & 11.4 & $85 \cdot 8$ & $40 \cdot 0-175$ & 11.5 & 78.0 & $23 \cdot 3-170$ & $15 \cdot 4$ & 78.0 & $44 \cdot 5-170$ & 0.02 \\
\hline Dried Fruit & 8.0 & $24 \cdot 1$ & $8.9-50 \cdot 0$ & $8 \cdot 3$ & $18 \cdot 8$ & $10 \cdot 8-32 \cdot 0$ & 0.87 & $5 \cdot 1$ & $13 \cdot 8$ & $7 \cdot 8-26 \cdot 8$ & 9.1 & 23.0 & $15 \cdot 3-46 \cdot 9$ & $10 \cdot 1$ & 21.2 & $9.4-46.9$ & $<0.001$ \\
\hline \multirow[t]{4}{*}{ Fruit Juice§ } & $19 \cdot 3$ & 150 & $150-150$ & $17 \cdot 3$ & 150 & $150-150$ & 0.17 & $16 \cdot 7$ & 150 & $150-150$ & $19 \cdot 4$ & 150 & $150-150$ & $18 \cdot 6$ & 150 & $150-150$ & 0.41 \\
\hline & \multicolumn{13}{|c|}{ BMI $\left(\mathrm{kg} / \mathrm{m}^{2}\right) \dagger$} & & & & \\
\hline & \multicolumn{3}{|c|}{$<18.5$} & \multicolumn{3}{|c|}{$18.5-24.99$} & & \multicolumn{3}{|c|}{ 25.0-29.99 } & \multicolumn{3}{|c|}{$\geq 30.0$} & & & & \\
\hline & $\%$ & Median & IQR & $\%$ & Median & IQR & & $\%$ & Median & IQR & $\%$ & Median & IQR & & & & $P^{*}$ \\
\hline Total Fruit without Juice & $37 \cdot 3$ & 219 & $164-343$ & 51.5 & 170 & $81 \cdot 0-290$ & & $45 \cdot 6$ & 188 & $150-315$ & $42 \cdot 2$ & 164 & $75 \cdot 0-262$ & & & & 0.02 \\
\hline Total Fruit including Juiceł & 41.8 & 179 & $152-384$ & 59.9 & 169 & 143-296 & & 53.7 & 174 & $150-315$ & $51 \cdot 0$ & 162 & $94 \cdot 0-274$ & & & & 0.01 \\
\hline Citrus Fruit & $9 \cdot 0$ & $15 \cdot 7$ & $15 \cdot 7-131$ & $10 \cdot 7$ & 92.8 & $75 \cdot 0-150$ & & $10 \cdot 6$ & 131 & $75 \cdot 0-193$ & 11.8 & 99.0 & $75 \cdot 0-193$ & & & & 0.10 \\
\hline Pome Fruit & 14.9 & 164 & $37 \cdot 9-164$ & $23 \cdot 2$ & 164 & $153-188$ & & 24.4 & 164 & $164-188$ & $19 \cdot 7$ & 164 & $153-188$ & & & & 0.06 \\
\hline Tropical Fruit & 7.5 & $51 \cdot 0$ & $51 \cdot 0-190$ & 9.9 & 41.9 & $18 \cdot 0-159$ & & $7 \cdot 3$ & 73.5 & $23 \cdot 1-367$ & $6 \cdot 3$ & 83.3 & $25 \cdot 1-367$ & & & & 0.54 \\
\hline Berries & $13 \cdot 4$ & $36 \cdot 0$ & $27 \cdot 4-139$ & 8.8 & 38.8 & $24 \cdot 0-110$ & & $6 \cdot 7$ & $36 \cdot 8$ & $19.0-88.0$ & $3 \cdot 0$ & 83.3 & $24 \cdot 0-159$ & & & & 0.06 \\
\hline Stone Fruit & 7.5 & 145 & $18 \cdot 3-165$ & 6.6 & 145 & $60 \cdot 0-217$ & & 4.9 & 145 & $75 \cdot 0-176$ & 7.1 & 151 & $83 \cdot 3-210$ & & & & 0.52 \\
\hline Other Fruit & 17.9 & $68 \cdot 8$ & $13 \cdot 9-100$ & $13 \cdot 9$ & 85.0 & $27 \cdot 0-175$ & & $12 \cdot 3$ & 136 & $33 \cdot 3-160$ & $10 \cdot 7$ & 78.0 & $44 \cdot 0-170$ & & & & 0.44 \\
\hline Dried Fruit & 4.5 & $50 \cdot 0$ & $50 \cdot 0-150$ & $10 \cdot 2$ & $18 \cdot 8$ & $12 \cdot 7-40 \cdot 2$ & & 8.2 & $24 \cdot 1$ & $9.4-46.9$ & $4 \cdot 1$ & 8.5 & $4 \cdot 2-24 \cdot 0$ & & & & 0.02 \\
\hline Fruit Juice§ & 13.4 & 150 & $150-150$ & 20.0 & 150 & $150-150$ & & $17 \cdot 3$ & 150 & $150-150$ & $15 \cdot 3$ & 150 & $150-150$ & & & & 0.42 \\
\hline
\end{tabular}

${ }^{*}$ From Kruskal-Wallis test on per capita intakes; significant $P$ values indicated in bold font

tn 2080, as 317 participants did not have a measured weight and height for calculation of BMI values.

flncluding fruit juice, up to 1 serving ( $125 \mathrm{ml}$ or $1 / 2$ cup) 


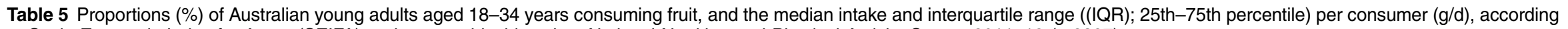
to Socio-Economic Index for Areas (SEIFA) and geographical location, National Nutrition and Physical Activity Survey 2011-12 ( $n$ 2397)

\begin{tabular}{|c|c|c|c|c|c|c|c|c|c|c|c|c|c|c|c|c|}
\hline & \multicolumn{16}{|c|}{ SEIFA } \\
\hline & \multicolumn{3}{|c|}{ Lowest $20 \%$} & \multicolumn{3}{|c|}{ Second quintile } & \multicolumn{3}{|c|}{ Third quintile } & \multicolumn{3}{|c|}{ Fourth quintile } & \multicolumn{3}{|c|}{ Highest $20 \%$} & \multirow[b]{2}{*}{$P^{*}$} \\
\hline & $\%$ & Median & IQR & $\%$ & Median & IQR & $\%$ & Median & IQR & $\%$ & Median & IQR & $\%$ & Median & IQR & \\
\hline Total Fruit without Juice & 37.3 & 184 & $131-303$ & 51.5 & 175 & $107-334$ & $45 \cdot 6$ & 188 & $125-280$ & $42 \cdot 2$ & 185 & $105-333$ & $51 \cdot 1$ & 184 & $102-276$ & 0.001 \\
\hline Total Fruit including Juice $†$ & 41.8 & 166 & $143-295$ & 59.9 & 164 & $150-314$ & 53.7 & 186 & $150-304$ & 51.0 & 181 & $150-303$ & $62 \cdot 1$ & 164 & $143-306$ & $<0.001$ \\
\hline Citrus Fruit & 9.0 & $75 \cdot 0$ & $58 \cdot 0-225$ & 10.7 & 131 & $75 \cdot 0-193$ & $10 \cdot 6$ & 131 & $75 \cdot 0-193$ & 11.8 & $75 \cdot 0$ & $75 \cdot 0-150$ & $12 \cdot 3$ & 91.9 & $75 \cdot 0-150$ & 0.14 \\
\hline Pome Fruit & $14 \cdot 9$ & 164 & $153-188$ & 23.2 & 164 & $153-188$ & $24 \cdot 4$ & 164 & $153-188$ & $19 \cdot 7$ & 173 & $153-188$ & $22 \cdot 3$ & 164 & $153-188$ & 0.10 \\
\hline Tropical Fruit & 7.5 & 73.5 & $10 \cdot 8-294$ & 9.9 & $72 \cdot 0$ & $9 \cdot 8-190$ & 7.3 & $40 \cdot 0$ & $23.3-73.9$ & $6 \cdot 3$ & 62.9 & $19 \cdot 2-367$ & 9.7 & 51.0 & $16 \cdot 4-159$ & 0.04 \\
\hline Berries & 13.4 & $36 \cdot 0$ & $20 \cdot 8-83 \cdot 3$ & 8.8 & 96.0 & $44 \cdot 3-139$ & 6.7 & 38.5 & $23 \cdot 3-72 \cdot 0$ & 3.0 & 43.4 & $24 \cdot 0-110$ & $11 \cdot 2$ & $30 \cdot 7$ & $18 \cdot 0-66.0$ & 0.003 \\
\hline Stone Fruit & 7.5 & 151 & $83 \cdot 3-165$ & 6.6 & 145 & $40 \cdot 0-201$ & 4.9 & 145 & $75 \cdot 0-151$ & 7.1 & 151 & $60 \cdot 0-210$ & $7 \cdot 1$ & 118 & $46 \cdot 4-290$ & 0.23 \\
\hline Other Fruit & 17.9 & 121 & $44 \cdot 0-218$ & 13.9 & 78.0 & $26 \cdot 0-156$ & $12 \cdot 3$ & $75 \cdot 0$ & $21 \cdot 8-160$ & $10 \cdot 7$ & 126 & $62 \cdot 9-170$ & $16 \cdot 5$ & 78.0 & $44 \cdot 0-204$ & 0.02 \\
\hline Dried Fruit & 4.5 & 8.0 & $3.5-13.5$ & $10 \cdot 2$ & $26 \cdot 8$ & $10 \cdot 8-50.0$ & 8.2 & $20 \cdot 1$ & $6 \cdot 7-35 \cdot 0$ & $4 \cdot 1$ & 21.6 & $16 \cdot 3-50 \cdot 0$ & 8.7 & $26 \cdot 1$ & $12 \cdot 7-41 \cdot 7$ & 0.02 \\
\hline Fruit Juiceł & 13.4 & 150 & $150-150$ & 20.0 & 150 & $150-150$ & $17 \cdot 3$ & 150 & $150-150$ & $15 \cdot 3$ & 150 & $150-150$ & $24 \cdot 7$ & 150 & 150-150 & $<0.001$ \\
\hline
\end{tabular}

\begin{tabular}{|c|c|c|c|c|c|c|c|c|c|c|}
\hline & \multicolumn{3}{|c|}{ City } & \multicolumn{3}{|c|}{ Inner regional } & \multicolumn{3}{|c|}{ Outer regional/remote } & \multirow[b]{2}{*}{$P^{*}$} \\
\hline & $\%$ & Median & IQR & $\%$ & Median & IQR & $\%$ & Median & IQR & \\
\hline Total Fruit without Juice & $49 \cdot 1$ & 188 & 128-307 & $44 \cdot 1$ & 160 & $75 \cdot 0-215$ & $44 \cdot 8$ & 188 & $102-294$ & 0.02 \\
\hline Total Fruit including Juice† & 57.9 & 170 & $150-304$ & $51 \cdot 0$ & 164 & 113-285 & 51.9 & 187 & 150-309 & 0.01 \\
\hline Citrus Fruit & 11.9 & 131 & $75 \cdot 0-193$ & 8.3 & 93.0 & $75 \cdot 0-150$ & 11.0 & 131 & $65 \cdot 5-262$ & $0 \cdot 10$ \\
\hline Pome Fruit & $24 \cdot 3$ & 164 & 153-188 & $20 \cdot 1$ & 164 & $135-188$ & $20 \cdot 6$ & 164 & 153-188 & 0.06 \\
\hline Tropical Fruit & 8.0 & $51 \cdot 0$ & $16 \cdot 4-190$ & $8 \cdot 1$ & $23 \cdot 1$ & $14 \cdot 3-73.5$ & 9.9 & 56.6 & $40 \cdot 0-193$ & 0.54 \\
\hline Berries & $7 \cdot 6$ & 38.5 & $24 \cdot 0-101$ & $5 \cdot 1$ & $24 \cdot 0$ & $19 \cdot 0-114$ & $9 \cdot 6$ & $56 \cdot 6$ & $24 \cdot 0-80 \cdot 2$ & 0.06 \\
\hline Stone Fruit & 6.5 & 151 & $60 \cdot 0-217$ & $5 \cdot 1$ & 145 & $40 \cdot 0-210$ & $7 \cdot 2$ & 145 & $66 \cdot 0-151$ & 0.52 \\
\hline Other Fruit & 13.3 & 85.0 & $40 \cdot 0-170$ & $11 \cdot 0$ & 78.0 & $26 \cdot 4-156$ & 13.4 & 62.9 & $20 \cdot 8-221$ & 0.44 \\
\hline Dried Fruit & 8.5 & $19 \cdot 2$ & $9 \cdot 4-40 \cdot 2$ & 9.8 & $16 \cdot 7$ & $13 \cdot 4-51 \cdot 2$ & 4.5 & $20 \cdot 0$ & $17 \cdot 8-35 \cdot 6$ & 0.02 \\
\hline Fruit Juiceł & $18 \cdot 6$ & 150 & $150-150$ & 15.9 & 150 & $150-150$ & $19 \cdot 1$ & 150 & $150-150$ & 0.42 \\
\hline
\end{tabular}

${ }^{*}$ From Kruskal-Wallis test on per capita intakes; significant $P$ values indicated in bold font.

tIncluding fruit juice, up to 1 serving ( $125 \mathrm{ml}$ or $1 / 2$ cup).

fUp to 1 serving $(125 \mathrm{ml}$ or $1 / 2$ cup . 
Table 6 Proportions of Australian young adults aged 18-34 years consuming a low, medium and high variety of vegetable and fruit sub-categories, National Nutrition and Physical Activity Survey 2011-12 ( $n$ 2397)

\begin{tabular}{|c|c|c|c|c|c|c|}
\hline \multirow[b]{2}{*}{ Number of sub-categories consumed ${ }^{*}$} & \multicolumn{2}{|c|}{$\begin{array}{l}\text { 18-24-year-olds } \\
\text { ( } n \text { 780) }\end{array}$} & \multicolumn{2}{|c|}{$\begin{array}{l}\text { 25-29-year-olds } \\
\text { ( } n \text { 736) }\end{array}$} & \multicolumn{2}{|c|}{$\begin{array}{c}\text { 30-34-year-olds } \\
(n \text { 881) }\end{array}$} \\
\hline & $\%$ & $n$ & $\%$ & $n$ & $\%$ & $n$ \\
\hline \multicolumn{7}{|l|}{ Vegetables $†$} \\
\hline$<1$ & 26.9 & 210 & $19 \cdot 8$ & 146 & $21 \cdot 3$ & 188 \\
\hline $1-2$ (low) & 57.4 & 448 & $59 \cdot 2$ & 436 & 57.9 & 510 \\
\hline 3-4 (medium) & $15 \cdot 0 \S$ & 117 & $18 \cdot 9$ & 139 & $19 \cdot 6$ & 173 \\
\hline$\geq 5$ (high) & 0.6 & 5 & $2 \cdot 0$ & 15 & $1 \cdot 1$ & 10 \\
\hline \multicolumn{7}{|l|}{ Fruitł } \\
\hline$<1$ & 67.4 & 526 & $62 \cdot 6$ & 461 & $59 \cdot 8$ & 527 \\
\hline 1 (low) & 24.2 & 189 & 28.0 & 206 & 29.7 & 262 \\
\hline 2 (medium) & 6.7 & 52 & 8.4 & 62 & $8 \cdot 7$ & 77 \\
\hline$\geq 3$ (high) & 1.7 & 13 & 1.0 & 7 & 1.7 & 15 \\
\hline
\end{tabular}

${ }^{*}$ Consumption of a category defined as eating at least half a serving of fruit or vegetable within the category ( $\geq 37.5 \mathrm{~g}$ of vegetables or $\geq 75 \mathrm{~g}$ of fruit). †Excluding fried potatoes.

$\ddagger$ Excluding fruit juice and dried fruit.

$\S$ Significant difference in proportion scoring $\geq 3$ for vegetable variety score by age using post hoc $X^{2}$ analysis $(z=3 \cdot 0, P<0.008$, Bonferroni-corrected $P$ value $)$.

(5 servings) minimum recommended daily intake. Approximately $15 \%$ of the young adults consumed $\geq 5$ servings of vegetables and $\geq 2$ servings of fruit on the day prior to recall.

\section{Fruit and vegetable variety}

The variety of fruits and vegetables consumed by the respondents is presented in Table 6. Less than a quarter of population surveyed reported consuming 3-4 different vegetable categories on the day prior to the dietary recall. Among those who consumed vegetables, intake of starchy vegetables was high (approximately 1.2 servings) but consumption of the green and brassica group was less than half a serving (Table 2). A large proportion of the young adults consumed $<1$ type of fruit, with citrus, pome and stone fruits eaten the most among fruit consumers (Table 4). There were no differences in fruit variety (consuming $\geq 2$ categories) by age or gender. However, those aged 18-24 years had the lowest vegetable variety score $(P=0 \cdot 01)$, with no differences by gender.

\section{Analysis by meal occasion}

Differences in fruit and vegetable intake were observed across meal occasions $(P<0 \cdot 001)$. The highest mean intake of vegetables occurred at dinner (131 (SD 212) g, 1.75 servings), followed by lunch (64.7 (sD 101) g). Less than a quarter of a serving of vegetables was reported at breakfast (12.5 (sD 52.2) g) and as snacks (15.5 (sD 64.5) g). Fruit consumption was highest between main meals with almost half a serving consumed as snacks (68.9 (SD 128) g). Table 7 demonstrates the differences in proportions consuming fruits and vegetables per meal occasion grouped according to the number of servings consumed throughout the day. Those consuming $>5$ vegetable servings daily had the highest proportion of consumers across all meals $(P<0 \cdot 001)$. Additionally, a larger proportion of respondents who consumed $>2$ fruit servings/d reported intake of fruit as a snack and at lunch compared with those consuming $\leq 1$ serving/d $(P<0 \cdot 001$; Table 7$)$.

\section{Associations between fruit and vegetable intake and lifestyle, anthropometry and sociodemographic variables: linear modelling}

Table 8 shows the associations between fruit and vegetable intake and sociodemographic and lifestyle factors. A positive association was observed between age and fruit and vegetable intake $(P=0.002$, excluding juice; $P=0.003$ including juice; $P<0 \cdot 001$, vegetables). When controlling for energy males consumed less vegetables than females $(P=0.002)$. There were no associations found between BMI and intake (Table 8 ). While the removal of underreporters increased $\beta$ values positively, the associations remained non-significant. Living in outer regional and remote areas was associated with the lowest fruit intake ( $P=0 \cdot 01$, excluding juice). No associations were found between intake and SEIFA categories.

\section{Discussion}

The present secondary analysis of the 2011-12 NNPAS data confirms that fruit and vegetable intakes of young adults aged 18-34 years are suboptimal. The combined mean fruit and vegetable intake of the surveyed sample $(328 \mathrm{~g} / \mathrm{d})$ fell short of the WHO standard, which recommends $400-500 \mathrm{~g}$ of fruits and vegetables daily for prevention of chronic disease risk ${ }^{(12)}$ and aligns with previous reports on the global inadequacy of population intakes ${ }^{(52)}$. Most Australian young adults also failed to consume a variety of fruits and vegetables, with those in the youngest age group (18-24 years) reporting the lowest intakes and variety. Analyses by sociodemographic variables revealed that males may need more support than females to improve intake as well as those in regional areas who have 
Table 7 Proportions (\%) of Australian young adults aged 18-34 years consuming vegetables and fruits per meal occasion (breakfast, lunch, dinner and snacks), grouped according to the number of servings consumed throughout the day, National Nutrition and Physical Activity Survey 2011-12 ( $n$ 2397)

\begin{tabular}{|c|c|c|c|c|c|c|c|}
\hline \multirow[b]{2}{*}{ Meal occasion } & \multicolumn{6}{|c|}{ Vegetables } & \multirow[b]{2}{*}{$P^{*}$} \\
\hline & $\begin{array}{c}\leq 75 \mathrm{~g} / \mathrm{d} \\
\leq 1 \text { serving } / \mathrm{d} \\
(n \text { 490) }\end{array}$ & $\begin{array}{c}76-150 \mathrm{~g} / \mathrm{d} \\
\leq 2 \text { servings/d } \\
(n 565)\end{array}$ & $\begin{array}{c}151-225 \mathrm{~g} / \mathrm{d} \\
\leq 3 \text { servings/d } \\
(n \text { 305) }\end{array}$ & $\begin{array}{c}226-300 \mathrm{~g} / \mathrm{d} \\
\leq 4 \text { servings/d } \\
(n \text { 283) }\end{array}$ & $\begin{array}{c}301-375 \mathrm{~g} / \mathrm{d} \\
\leq 5 \text { servings/d } \\
(n 182)\end{array}$ & $\begin{array}{c}>375 \mathrm{~g} / \mathrm{d} \\
>5 \text { servings/d } \\
(n \text { 360) }\end{array}$ & \\
\hline Breakfast & 3.5 & $6 \cdot 0$ & $10 \cdot 8$ & $11 \cdot 7$ & 11.5 & 19.7 & $<0.001$ \\
\hline Lunch & $47 \cdot 8$ & 58.9 & 64.9 & 67.5 & 78.6 & $71 \cdot 1$ & $<0.001$ \\
\hline Dinner & $67 \cdot 8$ & 83.2 & $90 \cdot 2$ & 91.2 & 93.4 & 95.8 & $<0.001$ \\
\hline Snacksł & $12 \cdot 2$ & $12 \cdot 4$ & $15 \cdot 4$ & $20 \cdot 1$ & $19 \cdot 8$ & $25 \cdot 3$ & $<0.001$ \\
\hline
\end{tabular}

\begin{tabular}{|c|c|c|c|c|}
\hline \multirow[b]{2}{*}{ Meal occasion } & \multicolumn{3}{|c|}{ Fruit† } & \multirow[b]{2}{*}{$P^{*}$} \\
\hline & $\begin{array}{c}\leq 150 \mathrm{~g} / \mathrm{d} \\
\leq 1 \text { serving/d } \\
(n 402)\end{array}$ & $\begin{array}{c}151-300 \mathrm{~g} / \mathrm{d} \\
\leq 2 \text { servings/d } \\
(n \text { 479) }\end{array}$ & $\begin{array}{c}>300 \mathrm{~g} / \mathrm{d} \\
>2 \text { servings/d } \\
(n 261)\end{array}$ & \\
\hline Breakfast & $31 \cdot 1$ & 22.5 & $31 \cdot 8$ & 0.01 \\
\hline Lunch & $16 \cdot 4$ & $15 \cdot 2$ & $27 \cdot 6$ & $<0.001$ \\
\hline Dinner & $18 \cdot 2$ & $10 \cdot 9$ & $15 \cdot 3$ & 0.01 \\
\hline Snacksł & $46 \cdot 3$ & 78.7 & 81.2 & $<0.001$ \\
\hline
\end{tabular}

${ }^{\star}$ From $X^{2}$ analysis of differences in proportions of persons consuming vegetables/fruits at each meal according to categories of servings consumed; significant $P$ values indicated in bold font.

†Excluding fruit juice.

†Snacks included all foods consumed between main meals.

Table 8 Linear regression results: factors associated with vegetable and fruit intake among Australian young adults aged 18-34 years, National Nutrition and Physical Activity Survey 2011-12 ( $n$ 2397)

\begin{tabular}{|c|c|c|c|}
\hline Sociodemographic variable & $\begin{array}{l}\text { Vegetables } \\
\beta \text { coefficient }^{*}\end{array}$ & $\begin{array}{l}\text { Fruit (excluding juice) } \\
\quad \beta \text { coefficient }^{*}\end{array}$ & $\begin{array}{l}\text { Fruit (including juice) } \\
\quad \beta \text { coefficient }^{\star}\end{array}$ \\
\hline Age group (years) & $F=10.3, P<0.001$ & $F=6 \cdot 1, P=0.002$ & $F=6.0, P=0.003$ \\
\hline $18-24^{\mathrm{R}}$ & 0.0 & 0.0 & 0.0 \\
\hline $25-29$ & $49 \cdot 0$ & 9.4 & 11.9 \\
\hline $30-34$ & 38.5 & $27 \cdot 0$ & $28 \cdot 3$ \\
\hline Gender & $F=9.3, P=0.002$ & $F=1.2, P=0.28$ & $F=0.003, P=0.95$ \\
\hline Male $^{R}$ & 0.0 & 0.0 & 0.0 \\
\hline Female & 31.6 & $7 \cdot 2$ & 0.4 \\
\hline BMI $\left(\mathrm{kg} / \mathrm{m}^{2}\right) \ddagger$ & $F=0.7, P=0.5$ & $F=1.6, P=0.2$ & $F=2.3, P=0.08$ \\
\hline$<18.5^{\mathrm{R}}$ & -0.3 & -20.8 & $-27 \cdot 3$ \\
\hline $18.5-24.99$ & 0.0 & 0.0 & 0.0 \\
\hline $25 \cdot 0-29.99$ & -18.0 & 0.7 & -4.2 \\
\hline$\geq 30.0$ & $-15 \cdot 3$ & $-24 \cdot 0$ & $-31 \cdot 8$ \\
\hline Socio-Economic Index for Areas (SEIFA) & $F=0.8, P=0.5$ & $F=0.4, P=0.82$ & $F=2.0, P=0.09$ \\
\hline Lowest $20 \%{ }^{\mathrm{R}}$ & 0.0 & 0.0 & 0.0 \\
\hline Second quintile & $13 \cdot 1$ & -0.3 & $-5 \cdot 3$ \\
\hline Third quintile & 4.6 & $8 \cdot 3$ & 13.7 \\
\hline Fourth quintile & 14.9 & $8 \cdot 8$ & $19 \cdot 8$ \\
\hline Highest $20 \%$ & $24 \cdot 1$ & $3 \cdot 3$ & $13 \cdot 6$ \\
\hline Geographical location & $F=1.5, P=0.2$ & $F=4.4, P=0.01$ & $F=2.6, P=0.07$ \\
\hline City ${ }^{R}$ & 0.0 & 0.0 & 0.0 \\
\hline Inner regional & $16 \cdot 0$ & -26.4 & -21.6 \\
\hline Outer regional/remote & $-21 \cdot 1$ & -0.1 & $-2 \cdot 4$ \\
\hline
\end{tabular}

*Beta coefficients represent the adjusted mean difference between each subgroup and the reference group (R), based on per capita intake in grams ( $n$ 2397), after controlling for confounders including age, gender, BMI, SEIFA, geographical location, smoking status and alcohol intake.

†Under-reporters ( $n$ 386) excluded.

less access to a variety of fresh vegetables. These findings can inform policy and health promotion practice to effectively close the gap between current consumption levels and recommended intake.

Young adults consumed a mean of 0.9 and 2.7 servings of fruits and vegetables daily, respectively. This is higher than the ABS analysis for 19-30-year-olds (0.7 and 2.2 servings of fruits and vegetables) ${ }^{(53)}$, but includes all sources of fruits and vegetables using disaggregated data. Overall, vegetable intake of young adults may be slightly better than reported in previous analysis but is still well below recommendations, and therefore public health 
messages promoting fruit and vegetable consumption remain important.

Previous data collected in 1995 do not report intake of young adults separately; however, mean daily intake for those aged 19 years or over was 3.6 servings of vegetables and approximately 1 serving of fruit ${ }^{(36)}$. While the food items, classification of fruits and vegetables and method of analyses differed between the surveys, it is evident that intake of fruits and vegetables remains poor and is worsening. Thus, immediate action is required to assist this generation of adults to improve their intake.

Despite literature indicating that access to a variety of fruits and vegetables is lower and costs are higher in regional areas of Australia ${ }^{(39,54-56)}$, no differences in intake were observed between geographical locations. However, those within regional locations reported consuming more starchy vegetables and less of the 'others' category. As fruits and vegetables are highly perishable, the costs of transportation to remote areas are high and with desert climates, water shortages and soil prohibiting local production in some areas ${ }^{(57)}$, it is not surprising that young adults in isolated rural areas consume less perishable vegetables. To address this, social marketing campaigns could focus on the promotion of nutritionally equivalent frozen and low-sugar and low-sodium canned fruits and vegetables as a means of increasing variety at low cost, particularly in regional areas. Examples include frozen berries or canned beans, tomatoes and mushrooms.

Studies in Australia have explored differences in fruit and vegetable intake by SES. While Giskes et al. identified lower intakes among adolescents living lower-SES $\operatorname{areas}^{(35)}$, and the New South Wales population health survey results (2014) showed that fewer people in disadvantaged areas met fruit and vegetable recommendations ${ }^{(58)}$, no studies have specifically looked at young adults. The present analysis found no differences in mean vegetable intake of young adults by SEIFA quintile. However, among the higher SEIFA group there was a trend towards greater consumption of the 'other vegetables', such as mushrooms and avocado, which tend to be more expensive. It may be worthwhile to run local rather than national campaigns that address the specific barriers relevant to fruit and vegetable intake for the population within their area of residence. With the perceived cost of vegetables identified as a significant barrier to intake among young adults ${ }^{(28,59)}$, campaigns could focus on budgeting for the inclusion of fruits and vegetables, particularly for lower SEIFA groups. Furthermore, previous research has indicated that there are no significant differences in knowledge of fruit and vegetable recommendations between socio-economic groups; however, those from higher SES quintiles scored significantly higher in their ability to make healthier food choices ${ }^{(60)}$. This suggests the lower-SES areas may need extra support in translating knowledge into behaviour.
The analysis of patterns of fruit intake by SEIFA group revealed that while the lowest intake was recorded for those in the lowest SEIFA quintile, the highest intake of fruit juice was among those of the top SEIFA group. These results contrast what is seen in the USA, where the highest juice consumption is reported among those of lower $\mathrm{SES}^{(61)}$. Industry reports on the trend of commercial fruit juice consumption estimate an annual growth in revenue from juice sales of $9 \cdot 8 \%$ in Australia ${ }^{(62)}$. This proliferation of juice sales through outlets that offer 'designer' juices may be contributing to a trend for juice consumption among young adults of higher SEIFA. Previous research in Australia highlighted that such juices were seen as a fashion accessory by young adults ${ }^{(63)}$. Although fruit juice can assist in meeting the recommended two fruit servings daily, the higher sugar and lower fibre content of these beverages and ease of overconsumption indicate that intake should continue to be monitored and emphasis placed on increasing whole fruit consumption and replacing juice with water. This is particularly important considering fruit juice promotes weight gain over the long term ${ }^{(64)}$.

Overall, variety was poor among the young adults. Fruit consumers mainly reported intake of pome, citrus and stone fruit with lower intakes of berries and tropical fruit. Among vegetable consumers, intake of starchy vegetables was high but consumption of the green and brassica group was less than half a serving. While starchy vegetables contain carbohydrates (which provide energy) and some vitamins, green leafy and brassica vegetables are rich in folate which has been postulated to reduce the risk of cancer $^{(18)}$ and neural tube defects ${ }^{(65)}$. They are also a good source of phytochemicals, Fe and vitamin C. Our estimates of vegetable intake counted potatoes prepared without fat as a starchy vegetable but did not include fried potatoes as per the Australian dietary guidelines. Among consumers the median intake of fried potatoes ( 1.2 servings) was proportionally high compared with other vegetables.

Only $12 \%$ of the young adults surveyed consumed legumes. The consumption of legumes is of value, as they are a relatively inexpensive source of protein, Fe, fibre and micronutrients. Thus, promoting intake of these proteinand nutrient-rich vegetables to young adults can help to improve vegetable intake while also reducing the total cost of meals. Additionally, with previous research highlighting the effect of exposure to fruits and vegetables in the early years of life on intake and variety consumed in adulthood $^{(66)}$, continued work is needed to promote consumption in younger children with initiatives such as the Stephanie Alexander Kitchen Garden Program ${ }^{(67)}$.

To our knowledge, the current analysis is the first to examine fruit and vegetable intake by meal occasion. The findings demonstrated that vegetables are consumed mainly at dinner and lunch, with an opportunity to increase intake at breakfast and as snacks. Fruit consumption was highest between main meals with almost 
half a serving consumed as snack. Additionally, a greater proportion of respondents who met or exceeded the daily recommendations consumed fruits and vegetables throughout the day. Thus, public health practitioners should consider encouraging intake at all meals to increase the likelihood of reaching the recommended daily intake of fruits and vegetables.

Finally, the low level of fruit and vegetable intake within the young adult population is a concern considering the continued risk of overweight and obesity in this age group ${ }^{(68)}$. Given the cross-sectional nature of these data, it is not surprising that there was no association observed between BMI category and intake. Previous longitudinal studies have confirmed, however, that increasing vegetable intake is associated with a reduction in weight ${ }^{(69)}$, with a recent systematic review confirming that consumption of whole fruit can reduce the risk for long-term weight gain in middle-aged adults ${ }^{(64)}$. Thus, promoting vegetable and whole fruit intake to young adults, especially those of higher BMI, may be beneficial to weight maintenance in their transition into adulthood. Furthermore, given the additional benefits of increased fruit and vegetable intake in reducing the risk of cancer, CVD and all-cause mortality ${ }^{(70)}$, promoting increased intake in this young generation may reduce the future burden of chronic disease.

\section{Strengths and limitations}

As with most dietary assessment methods, the $24 \mathrm{~h}$ recall has some measurement error introduced by inaccurate recall or estimation of intake ${ }^{(71)}$. It is also important to note that those classified as 'non-consumers' on the day of the interview may not typically be non-consumers. Thus, one day recalls may not be a reflection of usual intake among individuals, but provide a good estimation and snapshot of consumption at a population level, allowing public health researchers to assess how intake changes over time. We also looked at the effect of under-reporting, with no significant effect found on associations.

A significant strength of our secondary analysis was the use of detailed intake data including fruits and vegetables consumed as part of any mixed dish, providing a more comprehensive estimation of intake. Future analysis could explore the major mixed-meal sources of fruits and vegetables.

\section{Conclusions}

Fruit and vegetable intake remains suboptimal for Australian young adults aged 18-34 years, with poorer intakes among 18-24-year-olds and males. Therefore, intensive efforts are warranted to effectively promote fruits and vegetables to this at-risk population group to increase intake as they transition into adulthood. The analyses documented herein highlight the specific opportunities for improving intake, namely supporting younger adults aged 18-24 years, with a focus on engaging males to increase vegetable intake, promoting fruits and vegetables at all meal occasions, with inclusion in mixed dishes, to increase likelihood of meeting daily requirements. For those in regional areas with limited access to a variety of fresh fruits and vegetables, canned and frozen options can be explored.

\section{Acknowledgements}

Financial support: This research received no specific grant from any funding agency in the public, commercial or notfor-profit sectors. M.N. and A.G. are supported by the Australian Post-graduate Award (APA) for doctoral studies. Conflict of interest: The authors have no financial or personal conflicts of interest to declare. Authorship: M.N., Z.S., A.R., A.G. and M.A.-F. contributed to the study design. K.M. provided statistical support and all authors contributed to data analysis and/or interpretation of the results. M.N. drafted the manuscript and all authors read and approved the final version of the manuscript. Ethics of buman subject participation: In keeping with the National Statement on Ethical Conduct in Human Research ${ }^{(72)}$, this research was exempt from review by the institutional review board as all data were de-identified. The surveys used to obtain these data were conducted under the Federal Census and Statistics Act $1905^{(41)}$.

\section{Supplementary material}

To view supplementary material for this article, please visit https://doi.org/10.1017/S1368980017001124

\section{References}

1. Ledoux T, Hingle M \& Baranowski T (2011) Relationship of fruit and vegetable intake with adiposity: a systematic review. Obes Rev 12, e143-e150.

2. Boffetta P, Couto E, Wichmann J et al. (2010) Fruit and vegetable intake and overall cancer risk in the European Prospective Investigation into Cancer and Nutrition (EPIC). J Natl Cancer Inst 102, 529-537.

3. Lunet N, Lacerda-Vieira A \& Barros H (2005) Fruit and vegetables consumption and gastric cancer: a systematic review and meta-analysis of cohort studies. Nutr Cancer 53, 1-10.

4. Steinmetz KA \& Potter JD (1996) Vegetables, fruit, and cancer prevention: a review. J Am Diet Assoc 96, 1027-1039.

5. Dauchet L, Amouyel P, Hercberg S et al. (2006) Fruit and vegetable consumption and risk of coronary heart disease: a meta-analysis of cohort studies. J Nutr 136, 2588-2593.

6. Ness AR \& Powles JW (1997) Fruit and vegetables, and cardiovascular disease: a review. Int J Epidemiol 26, 1-13.

7. Wang X, Ouyang Y, Liu J et al. (2014) Fruit and vegetable consumption and mortality from all causes, cardiovascular disease, and cancer: systematic review and dose-response meta-analysis of prospective cohort studies. BMJ 349, g4490.

8. Hu D, Huang J, Wang Y et al. (2014) Fruits and vegetables consumption and risk of stroke: a meta-analysis of prospective cohort studies. Stroke 45, 1613-1619. 
9. Appel LJ, Moore TJ, Obarzanek E et al. (1997) A clinical trial of the effects of dietary patterns on blood pressure. $N$ Engl J Med 336, 1117-1124.

10. Miura K, Greenland P, Stamler J et al. (2004) Relation of vegetable, fruit, and meat intake to 7-year blood pressure change in middle-aged men: the Chicago Western Electric Study. Am J Epidemiol 159, 572-580.

11. Bazzano LA, He J, Ogden LG et al. (2002) Fruit and vegetable intake and risk of cardiovascular disease in US adults: the first National Health and Nutrition Examination Survey Epidemiologic Follow-up Study. Am J Clin Nutr $\mathbf{7 6}$, 93-99.

12. World Health Organization (2003) Diet, Nutrition and the Prevention of Chronic Diseases. Report of a Joint WHO/FAO Expert Consultation. WHO Technical Report Series no. 916. Geneva: WHO.

13. French Government, Programme National Nutrition Santé (2002) Fruits et légumes: au moins 5 par jour. http://www. mangerbouger.fr/bien-manger/que-veut-dire-bien-manger127/les-9-reperes/fruits-et-legumes-au-moins-5-par-jour.html (accessed May 2016).

14. National Health Service, NHS Choices (2015) Live Well. 5 A DAY. http://www.nhs.uk/livewell/5aday/Pages/5ADAY home.aspx (accessed January 2017).

15. National Health and Medical Research Council (2011) Australian Dietary Guidelines Incorporating the Australian Guide to Healthy Eating - Providing the Scientific Evidence for Healthier Australian Diets. Canberra, ACT: NHMRC.

16. Gibson RS (2007) The role of diet- and host-related factors in nutrient bioavailability and thus in nutrient-based dietary requirement estimates. Food Nutr Bull 28, 1 Suppl. Int., S77-S100.

17. Van Duyn MAS \& Pivonka E (2000) Overview of the health benefits of fruit and vegetable consumption for the dietetics professional: selected literature. J Am Diet Assoc 100, 1511-1521

18. National Health and Medical Research Ccouncil (2011) A modelling system to inform the revision of the Australian Guide to Healthy Eating. https://www.eatforhealth.gov. $\mathrm{au} /$ sites/default/files/files/public_consultation/n55a_dietary_ guidelines_food_modelling_111216.pdf (accessed December 2015).

19. Rekhy R \& McConchie R (2014) Promoting consumption of fruit and vegetables for better health. Have campaigns delivered on the goals? Appetite 79, 113-123.

20. Cox DN, Anderson AS, Lean ME et al. (1998) UK consumer attitudes, beliefs and barriers to increasing fruit and vegetable consumption. Public Health Nutr 1, 61-68.

21. Pomerleau J, Lock K, McKee M et al. (2004) The challenge of measuring global fruit and vegetable intake. J Nutr 134, $1175-1180$

22. Hall JN, Moore S, Harper SB et al. (2009) Global variability in fruit and vegetable consumption. Am J Prev Med 36, 402-409.e5.

23. Casagrande SS, Wang Y, Anderson C et al. (2007) Have Americans increased their fruit and vegetable intake? The trends between 1988 and 2002. Am J Prev Med 32, $257-263$.

24. Serdula MK GC, Kettel-Khan L et al. (2004) Trends in fruit and vegetable consumption among adults in the United States: behavioral risk factor surveillance system, 19942000. Am J Public Health 94, 1014-1018.

25. Public Health England \& Food Standards Agency (2014) National Diet and Nutrition Survey: results from Years 1 to 4 (combined) of the rolling programme for 2008 and 2009 to 2011 and 2012. Chapter 8, Table 8. https://www.gov.uk/ government/statistics/national-diet-and-nutrition-survey-resultsfrom-years-1-to-4-combined-of-the-rolling-programme-for-2008and-2009-to-2011-and-2012 (accessed October 2015).
26. Pollard CM, Miller MR, Daly AM et al. (2008) Increasing fruit and vegetable consumption: success of the Western Australian Go for $2 \& 5^{\circledR}$ campaign. Public Health Nutr 11, 314-320.

27. Australian Bureau of Statistics (2012) 4364.0.55.001 Australian Health Survey: First Results, 2011-12. Canberra, ACT: ABS.

28. Dumbrell S \& Mathai D (2008) Getting young men to eat more fruit and vegetables: a qualitative investigation. Health Promot J Aust 19, 216-221.

29. Hartmann C, Dohle S \& Siegrist M (2014) Time for change? Food choices in the transition to cohabitation and parenthood. Public Health Nutr 17, 2730-2739.

30. Lee HA \& Park H (2015) Correlations between poor micronutrition in family members and potential risk factors for poor diet in children and adolescents using Korean National Health and Nutrition Examination Survey Data. Nutrients 7, 6346-6361.

31. Noar SM, Benac CN \& Harris MS (2007) Does tailoring matter? Meta-analytic review of tailored print health behavior change interventions. Psychol Bull 133, 673-693.

32. Rasmussen M, Krølner R, Klepp K-I et al. (2006) Determinants of fruit and vegetable consumption among children and adolescents: a review of the literature. Part I: Quantitative studies. Int J Behav Nutr Phys Act 3, 22.

33. Dobson A, Porteous J, McElduff P et al. (1997) Dietary trends: estimates from food supply and survey data. Eur J Clin Nutr 51, 193-198.

34. Magarey A, McKean S \& Daniels L (2006) Evaluation of fruit and vegetable intakes of Australian adults: the National Nutrition Survey 1995. Aust $N Z$ J Public Health 30, 32-37.

35. Giskes K, Turrell G, Patterson C et al. (2002) Socioeconomic differences in fruit and vegetable consumption among Australian adolescents and adults. Public Health Nutr 5, 663-669.

36. Australian Bureau of Statistics (1997) 4802.0 - National Nutrition Survey: Selected Highlights, Australia, 1995. Canberra, ACT: ABS.

37. Dean WR \& Sharkey JR (2011) Rural and urban differences in the associations between characteristics of the community food environment and fruit and vegetable intake. $J$ Nutr Educ Behav 43, 426-433.

38. Reicks M, Randall JL \& Haynes BJ (1994) Factors affecting consumption of fruits and vegetables by low-income families. J Am Diet Assoc 94, 1309-1311.

39. Landrigan T \& Pollard C (2010) Food Access and Cost Survey (FACS). http://www.public.health.wa.gov.au/cproot/ 4115/2/Food\%20Access\%20and\%20Costs\%20Survey\%202010. pdf (accessed March 2016).

40. Partridge SR, McGeechan K, Bauman A et al. (2016) Improved eating behaviours mediate weight gain prevention of young adults: moderation and mediation results of a randomised controlled trial of TXT2BFiT, mHealth program. Int J Behav Nutr Phys Act 13, 44.

41. Australian Bureau of Statistics (2013) 4363.0.55.001 Australian Health Survey: User's Guide, 2011-13. Canberra, ACT: ABS.

42. National Institutes of Health (2010) Trials use technology to help young adults achieve healthy weights. http:// www.nih.gov/news/health/nov2010/nhlbi-29.htm (accessed September 2015)

43. Australian Institute of Health (2007) Young Australians: their health and wellbeing (2007) Part 1: Background. http:// www.aihw.gov.au/WorkArea/DownloadAsset.aspx?id=6442 459815 (accessed January 2017).

44. Arnett JJ (2000) Emerging adulthood: a theory of development from the late teens through the twenties. Am Psychol 55, 469-480. 
45. Australian Bureau of Statistics (2013) 2011-13 National Nutrition Survey [confidentialised unit record file (CURF) on CD-ROM]. Canberra, ACT: ABS.

46. Food Standards Australia New Zealand (2011/13) Australian Food Supplement and Nutrient Database: Food Recipe File. http://www.foodstandards.gov.au/science/monitoring nutrients/ausnut/ausnutdatafiles/Pages/foodrecipe.aspx (accessed November 2015).

47. National Health Service, NHS Choices (2015) Rough guide Fruit and vegetable portion sizes. http://www.nhs.uk/livewell/5aday/documents/downloads/5aday_portion_guide. pdf (accessed January 2017).

48. NHLBI Obesity Education Initiative, National Heart, Lung, and Blood Institute, North American Association for the Study of Obesity, Expert Panel on the Identification, Treatment of Overweight and Obesity in Adults (2002) The Practical Guide: Identification, Evaluation, and Treatment of Overweight and Obesity in Adults. Bethesda, MD: National Heart, Lung, and Blood Institute.

49. Black A (2000) The sensitivity and specificity of the Goldberg cut-off for EI:BMR for identifying diet reports of poor validity. Eur J Clin Nutr 54, 395-404.

50. Rangan A, Allman-Farinelli M, Donohoe E et al. (2014) Misreporting of energy intake in the 2007 Australian Children's Survey: differences in the reporting of food types between plausible, under-and over-reporters of energy intake. J Hum Nutr Diet 27, 450-458.

51. Rennie KL, Coward A \& Jebb SA (2007) Estimating underreporting of energy intake in dietary surveys using an individualised method. Br J Nutr 97, 1169-1176.

52. Miller V, Yusuf S, Chow CK et al. (2016) Availability, affordability, and consumption of fruits and vegetables in 18 countries across income levels: findings from the Prospective Urban Rural Epidemiology (PURE) study. Lancet Glob Health 4, e695-e703.

53. Australian Bureau of Statistics (2013) Table 5.1: Mean Daily Food Intake, 4364.0.55.007. In 43640D0005_20112012 Australian Health Survey: Nutrition First Results - Foods and Nutrients, 2011-12. Canberra, ACT: ABS.

54. Harrison MS, Coyne T, Lee AJ et al. (2007) The increasing cost of the basic foods required to promote health in Queensland. Med J Aust 186, 9-14.

55. Chapman K, Kelly B, Bauman A et al. (2014) Trends in the cost of a healthy food basket and fruit and vegetable availability in New South Wales, Australia, between 2006 and 2009. Nutr Diet 71, 117-126.

56. Cancer Council NSW (2007) NSW healthy food basket cost, availability and quality survey. https://www.cancercouncil. com.au/576/cancer-prevention/diet-exercise/nutrition-policy/ food-costs/nsw-healthy-food-basket-2007/ (accessed June 2017).

57. Edwards F, Dixon J, Friel S et al. (2011) Climate change adaptation at the intersection of food and health. Asia Pac J Public Health 23, 2 Suppl., 91S-104S.

58. Centre for Epidemiology and Research (2014) Centre for Epidemiology and Research Health Statistics New South
Wales. Sydney, NSW: NSW Ministry of Health Health Statistics.

59. Hartman H, Wadsworth DP, Penny S et al. (2013) Psychosocial determinants of fruit and vegetable consumption among students in a New Zealand university. Results of focus group interviews. Appetite 65, 35-42.

60. Hendrie GA, Coveney J \& Cox D (2008) Exploring nutrition knowledge and the demographic variation in knowledge levels in an Australian community sample. Public Health Nutr 11, 1365-1371.

61. Drewnowski A \& Rehm CD (2015) Socioeconomic gradient in consumption of whole fruit and 100\% fruit juice among US children and adults. Nutr J 14, 3 .

62. IBISWorld (2016) Juice and Smoothie Bars in Australia: Market Research Report. http://www.ibisworld.com.au/ industry/juice-and-smoothie-bars.html (accessed March 2016).

63. Hattersley L, Irwin M, King L et al. (2009) Determinants and patterns of soft drink consumption in young adults: a qualitative analysis. Public Health Nutr 12, 1816-1822.

64. Hebden L, O'Leary F, Rangan A et al. (2015) Fruit consumption and adiposity status in adults: a systematic review of current evidence. Crit Rev Food Sci Nutr 57, 2526-2540.

65. De-Regil L, Fernández-Gaxiola A, Dowswell T et al. (2010) Effects and safety of periconceptional folate supplementation for preventing birth defects. Cochrane Database Syst Rev issue 10, CD007950.

66. Ramsay SA, Rudley M, Tonnemaker LE et al. (2016) A comparison of college students' reported fruit and vegetable liking and intake from childhood to adulthood. $J$ Am Coll Nutr 36, 28-37.

67. Block K, Gibbs L, Staiger PK et al. (2012) Growing community the impact of the Stephanie Alexander Kitchen Garden Program on the social and learning environment in primary schools. Health Educ Behav 39, 419-432.

68. Grech A \& Allman-Farinelli M (2016) Prevalence and period trends of overweight and obesity in Australian young adults. Eur J Clin Nutr 70, 1083-1085.

69. Bertoia M, Mukamal KJ, Cahill L et al. (2016) Changes in intake of fruits and vegetables and weight change in US men and women followed for up to 24 years: analysis from three prospective cohort studies. PLoS Med 13, e1001956.

70. Oyebode O, Gordon-Dseagu V, Walker A et al. (2014) Fruit and vegetable consumption and all-cause, cancer and CVD mortality: analysis of Health Survey for England data. J Epidemiol Community Health 68, 856-862.

71. Slimani N, Ferrari P, Ocke M et al. (2000) Standardization of the 24-hour diet recall calibration method used in the European prospective investigation into cancer and nutrition (EPIC): general concepts and preliminary results. Eur J Clin Nutr 54, 900-917.

72. National Health and Medical Research Council (2015) National Statement on Ethical Conduct in Human Research, 2007 (Updated May 2015). Canberra, ACT: NHMRC. 